\title{
Non-noble metal anode based dual-ion batteries: promising high-energy and low-cost energy storage devices
}

Zhiyong Tang

Lithium-ion batteries (LIBs), as one of the most commonly used electrochemical energy storage devices, have dominated today's markets for portable electronics and electric vehicles [1]. However, their energy density cannot yet satisfy the increasing requirement, so it is imperative for developing next-generation batteries with high energy density together with long cycling life and good safety.

In recent years, a novel type of battery called as dual-ion battery (DIB) has been received special attention owing to its wider voltage window, lower cost, and better safety compared with conventional LIB [2]. In a typical DIB, graphite is utilized as both the cathode and anode materials due to its intrinsic redox amphotericity, while lithium salts dissolved in organic electrolyte or ionic liquid are adopted as the electrolyte. During the charging process, anions and cations intercalate into the graphite cathode and graphite anode respectively, as the discharging process works reversely. Owing to the high operating potentials for the anion intercalation into graphite, the cut-off voltage of DIBs $(4.5-5.2 \mathrm{~V})$ is considerably higher than conventional LIBs (below $4.2 \mathrm{~V}$ ) [3], which is beneficial for increasing the energy density. However, the cycling stability of most reported DIBs is still unsatisfactory owing to the poor electrochemical stability of organic electrolyte under high voltage.

Recently, Prof. Yongbing Tang and co-workers from Shenzhen Institutes of Advanced Technology, Chinese Academy of Sciences, developed a novel and low-cost aluminum-graphite DIB (AGDIB) [4], using environmentally-friendly and low-cost aluminum foil as both the anode and current collector, graphite as the cathode material, and a specially designed $\mathrm{LiPF}_{6}$-contained carbonate as the electrolyte. The working mechanism is demonstrated to be as follows: upon charging, $\mathrm{PF}_{6}{ }^{-}$anions in the electrolyte intercalate into the graphite cathode, while the $\mathrm{Li}^{+}$cations deposit onto the aluminum anode to form an AlLi alloy. Impressively, the AGDIB was demonstrated to deliver a reversible capacity of $104 \mathrm{~mA} \mathrm{~h} \mathrm{~g}^{-1}$ (based on the mass of graphite $)$ at $2 \mathrm{C}\left(1 \mathrm{C}=100 \mathrm{~mA} \mathrm{~g}^{-1}\right)$ current rate, and a capacity retention of $88 \%$ after 200 cycles. As the $\mathrm{Al}$ foil acted as both the negative current collector and the negative active material, the AGDIB showed significantly reduced dead load and dead volume. Consequently, the AGDIB exhibited high packaged energy density of $\sim 220$ $\mathrm{W} \mathrm{h} \mathrm{kg}{ }^{-1}$ at a power density of $\sim 130 \mathrm{~W} \mathrm{~kg}^{-1}$, and $\sim 150 \mathrm{~W} \mathrm{~h}$ $\mathrm{kg}^{-1}$ at $\sim 1200 \mathrm{~W} \mathrm{~kg}^{-1}$, which were $\sim 50 \%$ higher than most commercial LIBs. While the AGDIB achieved high packaged energy density and high power density, the cycling stability was still needed to be improved because of the poor electrochemical stability of organic electrolyte under high voltage as well as the crack and pulverization problem of $\mathrm{Al}$ foil during cycling.

To solve this issue, Tang's group further utilized ionic liquid electrolyte to improve the cycling stability of the Al-graphite DIB [5]. Owing to the merits of negligible vapor pressure, nonflammability, excellent electrochemical stability over a wide voltage window, ionic liquid is suitable as the solvent for DIB with high working voltage. As a result, the Al-graphite DIB based on ionic liquid electrolyte showed an improved cycling stability with a reversible capacity of $\sim 98 \mathrm{~mA} \mathrm{~h} \mathrm{~g}^{-1}$ after 300 cycles at 0.5 $\mathrm{C}$ with negligible capacity decay. In their recent published work [6], they prepared a three-dimensional (3D) porous

CAS Key Laboratory of Nanosystem and Hierarchical Fabrication, CAS Center for Excellence in Nanoscience, National Center for Nanoscience and Technology, Beijing 100190, China

Email: zytang@nanoctr.cn 
$\mathrm{Al}$ foil coated with a uniform carbon layer ( $\mathrm{pAl} / \mathrm{C}$ ) as the anode instead of $\mathrm{Al}$ foil in the DIB to significantly improve the cycling life (Fig. 1). Significantly, the 3D porous structure of $\mathrm{Al}$ effectively released the mechanical stress caused by the volume change of $\mathrm{Al}$ during electrochemical cycling, and also shortened the ion diffusion length. The carbon layer also helped buffer the $\mathrm{Al}$ volume change, and alleviated undesirable surface reactions through solid-electrolyte interphase film formation. Therefore, owing to the synergistic effect of the porous and conductive structure of the $\mathrm{pAl} / \mathrm{C}$ anode, the DIB exhibited excellent long-term cycling stability of over 1000 cycles with a reversible capacity of $93 \mathrm{~mA} \mathrm{~h} \mathrm{~g}^{-1}$ at $2 \mathrm{C}$ with $89.4 \%$ retention of capacity. Of particular interest, the energy density of this DIB was estimated to be $204 \mathrm{~W} \mathrm{~h} \mathrm{~kg}^{-1}$ at a high power density of $3084 \mathrm{~W} \mathrm{~kg}^{-1}$ (charging/discharging within four minutes), which was two times as large as most commercial LIBs and represented the best performance of any reported DIBs.
Although LIBs have been widely used, the limited lithium resources seriously hindered the applications of LIBs in the field of large-scale energy storage systems. Sodium-ion batteries (SIBs) are thus considered to be a promising alternative to conventional LIBs for future large-scale commercial applications, thanks to the abundance and low cost of sodium resources and similar physical and chemical properties to lithium [7-9]. Combining SIB technique with DIB technique, Tang's group recently reported a novel tin-graphite DIB (named as Sn-G DIB) based on a sodium-ion electrolyte containing $\mathrm{NaPF}_{6}$ salt, using $\mathrm{Sn}$ foil directly as both the anode and current collector, and expanded graphite as the cathode [10]. It is worthy to note that the Sn-G DIB could work reversibly with high capacity in the sodium-ion electrolyte over a high voltage window of 2.0-4.8 V. This battery delivered $74 \mathrm{~mA} \mathrm{~h} \mathrm{~g}^{-1}$ at $2 \mathrm{C}$ current rate and kept stable for 400 cycles with $94 \%$ capacity retention and high Coulombic efficiency of $95 \%$. $\mathbf{a}$
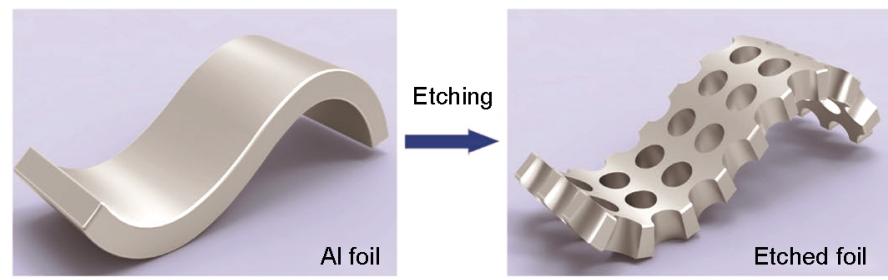

Etched foil
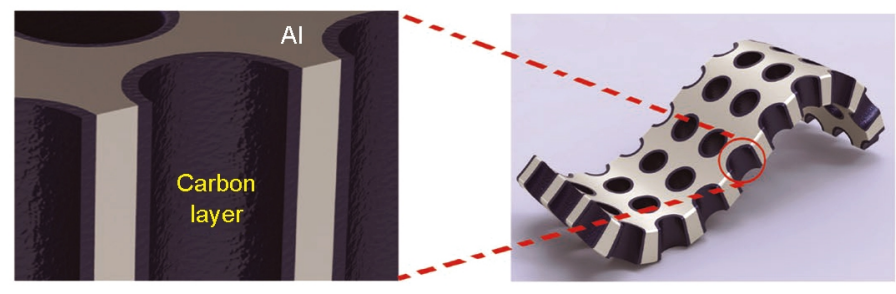

Carbonization
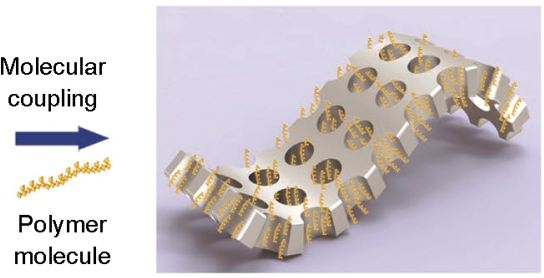

Solidification

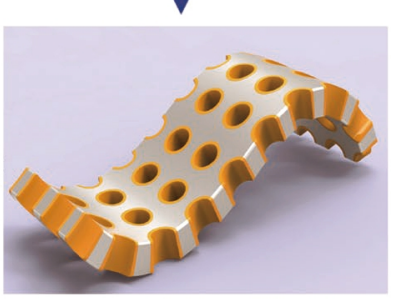

b

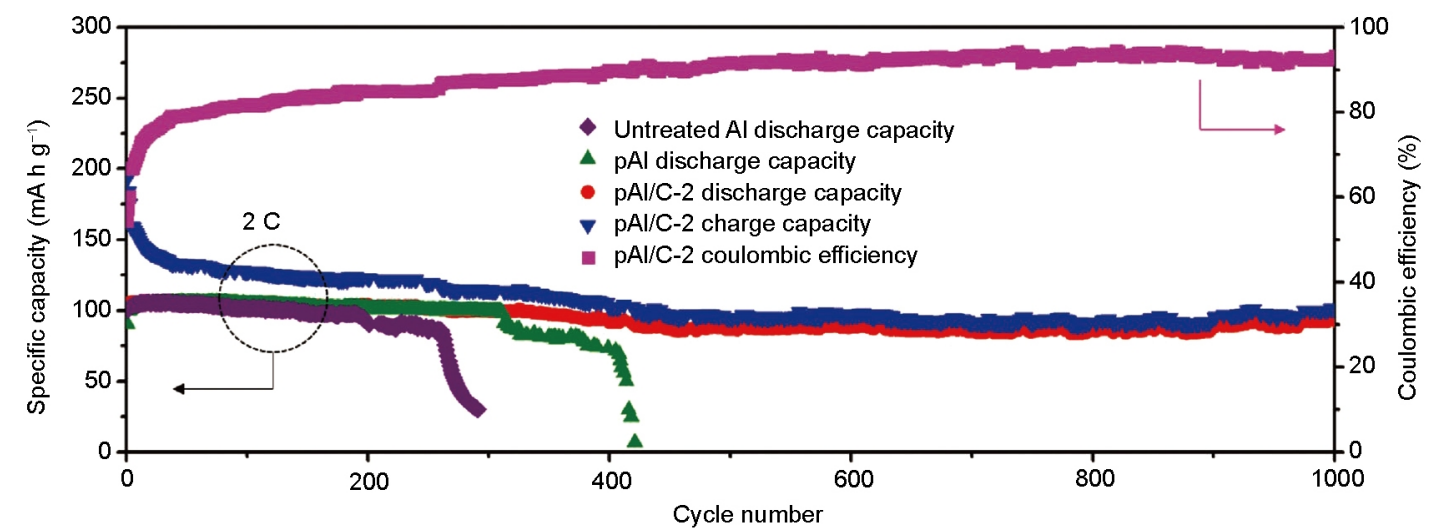

Figure 1 DIB based on carbon-coated porous $\mathrm{Al}$ foil anode. (a) Schematic illustration of the fabrication process to make the pAl/C anode material. (b) Long-term cycling performance of the pAl/C-G DIB at $2 \mathrm{C}$ for 1000 cycles with pAl and untreated Al foil as controls. Reprinted with permission from Ref. [6], Copyright 2016, Wiley. 
In addition, the packaged energy density of the Sn-G DIB was achieved to be $144 \mathrm{~W} \mathrm{~h} \mathrm{~kg}^{-1}$ at a power density of 150 $\mathrm{W} \mathrm{kg}^{-1}$, which was excitingly comparable with commercial LIBs.

Altogether, Prof. Yongbing Tang's continuous effort on DIBs utilizing the non-noble metal foil anodes with advantages of low-cost, high rate, high energy density, and long-term cycling capabilities show promising potential for industrial application in the energy field such as portable electronics and electric vehicles.

Received 17 February 2017; accepted 19 February 2017; published online 6 March 2017

1 Tarascon JM, Armand M. Issues and challenges facing rechargeable lithium batteries. Nature, 2001, 414: 359-367

2 Read JA, Cresce AV, Ervin MH, et al. Dual-graphite chemistry enabled by a high voltage electrolyte. Energ Environ Sci, 2014, 7: $617-620$

3 Rothermel S, Meister P, Schmuelling G, et al. Dual-graphite cells based on the reversible intercalation of bis(trifluoromethanesul- fonyl)imide anions from an ionic liquid electrolyte. Energ Environ Sci, 2014, 7: 3412-3423

4 Zhang X, Tang Y, Zhang F, et al. A novel aluminum-graphite dualion battery. Adv Energ Mater, 2016, 6: 1502588

5 Zhang F, Ji B, Tong X, et al. A dual-ion battery constructed with aluminum foil anode and mesocarbon microbead cathode via an alloying/intercalation process in an ionic liquid electrolyte. Adv Mater Interfaces, 2016, 3: 1600605

6 Tong X, Zhang F, Ji B, et al. Carbon-coated porous aluminum foil anode for high-rate, long-term cycling stability, and high energy density dual-ion batteries. Adv Mater, 2016, 28: 9979-9985

7 Massé RC, Uchaker E, Cao G. Beyond Li-ion: electrode materials for sodium- and magnesium-ion batteries. Sci China Mater, 2015, 58: $715-766$

8 Li W, Zeng L, Wu Y, et al. Nanostructured electrode materials for lithium-ion and sodium-ion batteries via electrospinning. Sci China Mater, 2016, 59: 287-321

9 Liu Z, Zhang Y, Zhao H, et al. Constructing monodispersed $\mathrm{MoSe}_{2}$ anchored on graphene: a superior nanomaterial for sodium storage. Sci China Mater, 2017, 60: 167-177

10 Sheng M, Zhang F, Ji B, et al. A novel tin-graphite dual-ion battery based on sodium-ion electrolyte with high energy density. Adv Energ Mater, 2016, 1601963 